\title{
Concept identification as a function of relevance of training and intertask instructions
}

GEDIMINAS NAMIKAS AND DONALD HARRIS. DEPARTMETT OF PSYCHOLOGY. WESTERV W.ISHIMUTON STATE (OLI.EGE, Bellingham. Wash. 98225

The salience of three types of verbal training was manipulated through instructions given before a concept identificution tusk. The l'rhal truining was either relevant $(R)$, irrelelant $(I R)$, or neutrul $(C$ ' with respect to the stimulus dimensions in the transfer task. It was found that: (a) $R$ training jacilitates concept identificution performance, and $(b) R$ and IR training respectively. facilitate and degrade concept identification performance after high salience instructions. but do not produce u differential effect on performance after low salience instructions.

\section{Problem}

A consistent facilitation of concept identification performance due to $R$ training has been reported in a series of studics (Gelland. 1958: Vamikas, 1962: Namikas, 1967), while the same studies hive found no corresponding degradation of performance due to IR training. The effect of IR training did not differ significantly from the effect of C training (Gelfand, 1958; Namikas. 1962: Namikas. 1967) or no training at all (Namikas. 1962).

In each of these experiments. the training stimuli were quite different from the stimuli used in the concept identification task. The training stimuli were either words that had to be memorized (Gelfand. 1958) or sorted into categories (Namikas, 1967). or simple (one dimensional) visual patterns (Namikas, 1962). The transfer stimuli in all cases were complex (multi-dimensional) visual patterns. Under these conditions, it may be assumed that the salience of the training task to the transfer task was rather low. As a result, the IR training experience may not be differentiated by the $S$ from the general background of irrelevant information characteristic of any experimental situation, as initially proposed by Namikas (1962) in a post hoc explanation of the lack of a decremental effect due to IR training.

The present study was designed to provide this type of differentiation through intertask instructions. It was hypothesized that instructing the $S$ that the training task was related to the transfer task would enhance the facilitative effect due to $R$ training and produce a degradation of performance due to IR training. Similarly, an instruction to the $S$ that the $(w)$ tasks were not related was expected to nullify the effect of $R$ training miking the performance of these Ss equal to the performance of the Ss who did indeed receive $I R$ training.

Method

Students from introductory psychology courses were assigned. in order of their appearance, to one of 24 groups. Each $S$ was seated in front of a four-compartment card sorting tray (15 in. long $\times 4$ in. high $\times 3-1 / 2$ in. wide). The front of each compartment was open on the $S$ 's side to facilitate the sorting of the cards by $S$.

During training, each $S$ sorted a set of stimultis cards ( 3 in. $x .3$ in.) on which a word had been typed in the center. A set of cards was made up of lour words that were duplicated 10 times. The words in two of the sets of cards referred to either relevant or irrelevant attributes of the transfer stimuli. The third set consisted of four neutral words and was used for the C training condition. The words were arranged in a random sequence with one restriction, namely. that a word would not follow itself. The pack of cards was arranged into a different sequence for every $S$.

The $S$ was told that the task was a card sorting task in which each of the cards would have to be sorted into one of the four compartments or categories. He was further told that both speed and accuracy of sorting were important. Verbal feedback was given after each sorting response and a correction procedure wats followed.

Immediately after the training session, the $E$ gave the $S$ another pack of stimulus cards to sort again into the four categories. The $S$ wiss instructed that he would have to perform the same type of task, but that each tin. he mude a. sorting response, he should wait for the verbal fecdlack from $E$ before he sorted the next card (a pilot study had indiated that. ollerwis. the $S$ tended in respond too fast for fecuback to be given after each response). Finally, $\mathrm{S}$ was told either that the training task was related to the new task or that it was not related.

The transfer stimuli were curds t.ıken from the Wisconsin Card Sorting Test. They varied only in two dimensions, however; color (red, green, blue, or yellow forms) ind form (circle. cross, triangle. or star). The number dimension was made sedundant by placing an opaque paper mask over all but one of the forms appearing on a particular card. An opaque mask was also placed on the cards that showed only one form, so that every card in the deck had in opaque mask of the same type.

All possible combinations of the two dinensions at four levels of each dimension were used $\left(4^{2}=16\right)$ and each stimulus was represented three times in the deck for a total of 48 cards. The stimulus cards were arranged in a different random sequence for every $S$ with no restrictions on the sequence. No correction was allowed in order to be consistent with the procedure used in previous studies. Each cutegorization response was followed by verbal feedback ("right" or "wrong"). All of the Ss went through the whole deck once.

The experimental design was a 2 by 3 by 2 by 3 orthogonal design. Two Es conducted the experiment. The Ss received either $\mathrm{R}, \mathrm{IR}$, or $\mathrm{C}$ training. They were told that the training task was either related or not related to the transfer task and then were given one of two concept identification prohlems (either color relevant, or form relevant). Five Ss were run in each of the 24 groups thus formed for a total of 120 Ss.

\section{Results and Discussion}

A total of 30 Ss were not able to verbalize the correct solution at the end of the experiment. The group which received IR training and then was given the high salience (related) instruction aciounted for 11 of these failures. A Chi-square test on the

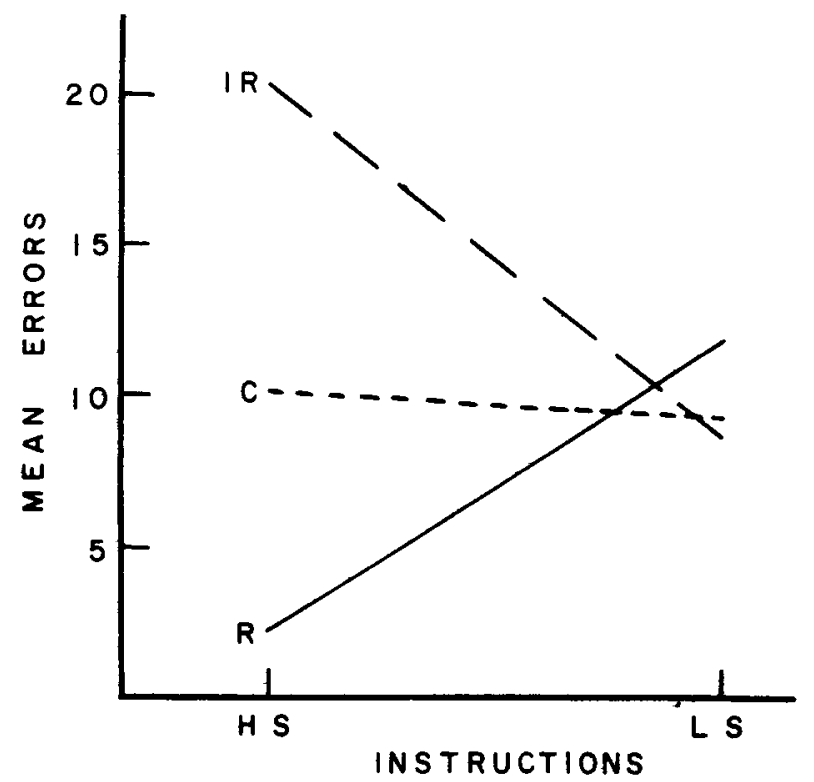

Fig. 1. Performance in a concept identification task as a function of relevant. control. or irrelevant verbal training and two types of intertask instructions. The instructions indicated that the training was related (high salience) or was not related (low salience) to the concept identification task. 
frequency of failures as a function of instructions and type of training, under the assumption that the number of failures should occur equally frequently for all conditions, was found to be highly significant (Chi-square $=10.800, \mathrm{df}=2, \mathrm{p}<.01$ ). The significant Chi-square could be accounted for mainly by two deviations from expectation, fewer than expected failures for the $\mathrm{R}$ training group which received high salience instructions (R-HS), and a larger than expected number of failures in the group receiving IR training followed by high salience instructions (IR-HS).

An analysis of variance on the errors-to-criterion data showed that the performance of the Ss on the concept identification task was not differentially influenced by the two Es conducting the study. Neither the E main effect, nor any of the interactions in which this variable entered was found to be statistically significant. As a consequence, the analysis was collapsed across this variable.

The problem in which color was relevant and form irrelevant ( $P$ II) was found to be significantly more difficult than the problem in which form was relevant and color irrelevant $(\mathrm{F}=6.96, \mathrm{df}=$ $1 / 108, \mathrm{p}<.005)$. The mean errors were: $\mathrm{P} \mathrm{I}=7.0, \mathrm{P} I \mathrm{II}=13.5$.

Type of training was the only other significant main effect $(F=$ 3.52 , $\mathrm{df}=2 / 108, .05>\mathrm{p}>.01$ ). A Multiple-Range test (Duncan, 1955 ) indicated that fewer errors were made after $\mathrm{R}$ training than after IR training (5\% level of confidence). The $\mathrm{C}$ group was not significantly different from the $\mathrm{R}$ or IR groups (mean errors were: $\mathrm{R}=7.0, \mathrm{C}=9.8, \mathrm{IR}=14.5$ ).

While the main effect of Instructions was not statistically significant, the interaction between type of training and instructions was highly significant $(F=6.97, \mathrm{df}=2 / 108, \mathrm{p}<.005)$. As may be seen in Fig. 1, the interaction is due to the differential effect on the performance of the IR and $R$ groups produced by the HS instructions. The performance of the $R$ group was enhanced by the HS instructions while the same instructions degraded the performance of the IR group. A comparison of the interaction means (Duncan, 1955) supported these conclusions. The analysis showed that the IR-HS group made significantly more errors to criterion than the R-HS ( $0.1 \%$ level of confidence), IR-LS ( $1 \%$ level of confidence), or R-LS, C-HS, and C-LS groups ( $5 \%$ level of confidence). In addition, $S$ s who received $R$ training followed by low salience (LS) instructions made significantly more errors than the R-HS group (5\% level of confidence).

The facilitative effect of $R$ training observed in the present study is in agreement with previous findings (Gelfand, 1958;
Namikas, 1962; Namikas, 1967). The effect, however, was attenuated by the instructions given to the $S$ after the training task. The R-LS group made significantly more errors than the R-HS group and its performance was not significantly different from the performance of the control groups (C-HS and C-LS) or the group which received IR training followed by low salience instructions (IR-LS).

The significant interaction between instructions and type of training, shown in Fig. 1, also supports and amplifies the findings of the previous studies with respect to the lack of effect of IR training on concept identification performance. The degrading effect of IR training was observed in the present experiment only after the S's attention was directed to the training experience as being salient to the concept identification task by means of misinformative instructions. Training experience which was perceived as not salient for the transfer task had no effect on the transfer performance irrespective of the actual relevance it had for the task, i.e., the R-LS and IR-LS did not differ significantly from each other or the C-LS group.

In the present study, the salience of the training task was established primarily by the intertask instructions. When no instructions of this type are provided, as in the previous experiments (Gelfand, 1958; etc.) it may be hypothesized that the salience of previous training is established by the informative feedback provided the $S$ during the transfer task. Since the informative feedback is consistently related to the attributes of the relevant dimensions, the salience of the $R$ training task is increased. Similarly, since the informative feedback is related to the attributes of the irrelevant dimensions at a chance level, the salience of the IR training task remains low, producing a transfer effect that is not significantly different from the effect of training on $\mathrm{C}$ dimensions.

\section{REFERENCES}

DUNCAN, D. B. Multiple range and multiple F tests. Biometrics, 1955, 11, $1-42$.

GELFAND, S. Effects of prior associations and task complexity upon the identification of concepts. Psychol Rep., 1958, 4, 567-574.

NAMIKAS, G. Effect of verbal pretraining on performance in a concept identification task. Unpublished doctoral dissertation, University of Wisconsin, Madison, 1962.

NAMIKAS, G. Concept identification as function of relevance of pre-training and percentage of informative feedback. Psychon. Sci. 1967, 8, 261-262. 